\title{
Estructuralismo, ficcionalismo, y la aplicabilidad de las matemáticas en ciencia*
}

\author{
Manuel Barrantes \\ Hamilton College
}

\begin{abstract}
Resumen: Este artículo tiene dos objetivos. El primero es una revisión de algunas de las preguntas más importantes de la filosofia de las matemáticas contemporánea, incluyendo: ¿Cuál es la naturaleza de los objetos matemáticos? ¿Cómo adquirimos conocimiento acerca de estos objetos? ¿Deben las afirmaciones matemáticas interpretarse de manera diferente a las afirmaciones ordinarias?, y finalmente, ¿cómo se explica la aplicabilidad de las matemáticas en la ciencia? El tema que guía estas reflexiones es el debate entre el realismo matemático y el antirrealismo. El segundo objetivo de este artículo es discutir cinco argumentos que usan la aplicabilidad de las matemáticas en ciencia para justificar el realismo matemático, y mostrar que ninguno de ellos consigue su objetivo. Con esta finalidad, distingo tres aspectos del problema de la aplicabilidad de las matemáticas: la (mera) utilidad de las matemáticas en ciencia; la inesperada utilidad de algunas teorías matemáticas; y la aparente indispensabilidad de las matemáticas en nuestras mejores teorías científicas, y en particular, en nuestras mejores explicaciones cientificas. Luego sostengo que ninguno de estos aspectos constituye una razón para adoptar el realismo matemático.

Palabras clave: realismo matemático; estructuralismo matemático; ficcionalismo; aplicabilidad; indispensabilidad; explicaciones matemáticas
\end{abstract}

\begin{abstract}
This article has two objectives. The first one is a review of some of the most important questions in the contemporary philosophy of mathematics, including: What is the nature of mathematical objects? How do we acquire knowledge about these objects? Should mathematical statements be interpreted differently than ordinary ones? And finally, how can we explain the applicability of mathematics in science? The topic that guides these reflections is the debate between mathematical realism and anti-realism. The second objective of this article is to discuss the arguments that use the applicability of mathematics in science to justify mathematical realism, and show that none of them reaches its objective. To this end, three aspects of the problem of the applicability of mathematics are distinguished: the (mere) utility of mathematics in science; the unexpected utility of some mathematical theories; and the apparent indispensability of mathematics in our best scientific theories, and in particular, in our best scientific explanations. Then I argue that none of these aspects constitutes a reason to adopt mathematical realism.
\end{abstract}

Keywords: mathematical realism; mathematical structuralism; factionalism; applicability; indispensability; mathematical explanations

\footnotetext{
* Quisiera agradecer los comentarios de la audiencia del High-Performance Computing Center de la Universidad de Stuttgart, donde este trabajo fue presentado por primera vez. Asimismo, agradezco a Alejandra Panizo por estimulantes conversaciones que me ayudaron a tomar la postura del platonismo matemático más seriamente.
} 


\section{Introducción: realismo en ontología y realismo en valor de verdad}

En la filosofia de las matemáticas existe una distinción entre el valor de verdad de las afirmaciones matemáticas y el estatuto ontológico de los objetos sobre los cuales dichas afirmaciones cuantifican. Stewart Shapiro presenta esta distinción en términos de realismo en ontología y realismo en valor de verdad. Realismo en ontología es la postura que sostiene que las afirmaciones matemáticas cuantifican sobre objetos reales, tales como números, conjuntos, etcétera. Estos objetos son "abstractos, acausales, indestructibles, eternos, no forman parte del espacio-tiempo" 1 y, además, existen objetivamente, es decir, "independientemente del matemático, su mente, su lenguaje, etc."2. Por otro lado, el realismo en valor de verdad sostiene que "las afirmaciones matemáticas tienen valor de verdad objetivo, independiente de las mentes, lenguajes, convenciones y más de los matemáticos"3.

El realismo en ontología y el realismo en valor de verdad no están necesariamente opuestos. Es fácil ver, por ejemplo, que, si uno cree que dichos objetos matemáticos existen, las afirmaciones acerca de ellos tendrán un valor de verdad objetivo. Así como la afirmación "las esmeraldas son verdes" es objetivamente verdadera en virtud del hecho de que las esmeraldas existen y son verdes, para alguien que suscribe ambos realismos la afirmación "existen infinitos números primos" también sería verdadera en virtud del hecho de que existen los números primos y hay una cantidad infinita de ellos ${ }^{4}$.

Por otro lado, uno puede ser antirrealista respecto de los objetos matemáticos y aun así otorgar un valor de verdad objetivo a las afirmaciones matemáticas. Por ejemplo, si se entiende a las matemáticas como un mero conjunto de afirmaciones sobre entidades ficcionales, entonces dichas afirmaciones matemáticas pueden tener un valor objetivo sin que esto implique que los objetos

\footnotetext{
1 Shapiro, S., "Philosophy of Mathematics and its Logic. Introduction", en: Shapiro, S. (ed.), The Oxford Handbook of Philosophy of Mathematics and Logic, Nueva York: Oxford University Press, 2005, p. 6. Todas las traducciones son mias.

Ibid.

3 Ibid.

4 Prueba de Euclides: si asumimos que p es el mayor número primo, y sumamos 1 al producto de todos los números primos, incluyendo $\mathrm{p}$, el resultado será un número primo mayor que $\mathrm{p}$. Por lo tanto, la noción de un mayor número primo es contradictoria. Por lo tanto, no existe un número primo que sea el mayor de todos.
} 
sobre los que estas afirmaciones cuantifican existan. Así, la afirmación "Frodo es el sobrino de Bilbo" es objetivamente verdadera respecto a la historia de $\mathrm{El}$ Señor de los Anillos sin que eso signifique que Bilbo y Frodo sean personas reales. Del mismo modo, la afirmación "existen infinitos números primos" es verdadera si se evalúa en el contexto de la historia del sistema de los números naturales, pero no necesariamente porque los números existan.

Dado que incluso los antirrealistas ontológicos pueden dar cuenta de la objetividad de las afirmaciones matemáticas, el foco principal en la filosofia de las matemáticas es el debate sobre el estatuto ontológico de los objetos matemáticos. Así, este debate ha opuesto a realistas matemáticos (que son en su mayoria realistas en valor de verdad y en ontología) y antirrealistas matemáticos (que son en su mayoría realistas en valor de verdad, pero antirrealistas en ontología).

En la primera parte de este artículo (secciones 2 y 3), introduzco las nociones de estructuralismo matemático ante rem y ficcionalismo matemático, que son las versiones de realismo y antirrealismo matemático sobre las que voy a discutir. Luego, explicaré por qué la aplicabilidad de las matemáticas en ciencia es importante en este debate. Seguidamente, en la segunda parte (secciones 4 y 5), discuto cinco argumentos que utilizan los diferentes aspectos de la aplicabilidad de las matemáticas en ciencia para justificar el realismo matemático (mera utilidad, efectividad "inesperada", e indispensabilidad) y muestro que ninguno de ellos cumple dicho objetivo.

Mi estrategia consiste, primero, en respetar la práctica matemática, es decir, en no proponer interpretaciones que vayan en contra de la forma como los matemáticos llevan a cabo su disciplina. Segundo, voy a criticar los argumentos realistas mínimamente, es decir, tratando de refutar el menor número posible de premisas. En ese sentido, mi estrategia es compatible con las siguientes posturas: naturalismo (la idea de que debemos mirar únicamente a la ciencia para saber qué existe); realismo científico (la idea de que la ciencia tiene como uno de sus objetivos brindar una descripción adecuada del mundo y que, en cierta medida, ha tenido éxito en esta tarea); indispensabilidad de las matemáticas (la idea de que nuestras mejores teorías científicas no serían tan exitosas si no hicieran uso de modelos matemáticos). Evidentemente, cada una de estas posturas puede y ha sido criticada. El punto principal de este artículo es que, incluso si uno asumiera que estas posturas son correctas, uno no tendría razones para ser realista matemático basado en la aplicabilidad de las matemáticas en ciencia. Esto, creo yo, fortalece mi argumento, pues sostengo que uno no tiene que entrar en los complicados y a veces enredados debates sobre 
el naturalismo, el realismo científico, etcétera, para refutar la idea de que la aplicabilidad de las matemáticas en ciencia justifica el realismo matemático.

\section{Realismo matemático y ficcionalismo matemático}

\subsection{Estructuralismo Matemático}

\section{a) Lo que nos números no pueden ser}

Uno de los principales problemas del realismo matemático, concebido como la postura de que los objetos matemáticos son acausales, abstractos y completamente separados del mundo físico (llamemos a esta postura "platonismo matemático puro"), es la pregunta sobre cuál puede ser la naturaleza de estos objetos matemáticos. Es conocida la preocupación de Gottlob Frege (1983-1965) acerca de que definir un número "n" como la propiedad compartida por todos los conjuntos de "n" miembros no informa acerca de qué cosa es el número "n" específicamente. ¿Puede el emperador Julio César ser el número "n"? Frege no tenía una respuesta definitiva a este problema (el llamado "problema de Julio César").

Más recientemente, Paul Benacerraf ${ }^{5}$ señaló que los números no pueden ser objetos. Existen diferentes teorias de conjuntos, ampliamente aceptadas, que definen a los números de diferentes formas. Por ejemplo, el número 3 es representado en la teoría de conjuntos de John von Neumann como $\{\varnothing,\{\varnothing\},\{\varnothing$, $\{\varnothing\}\}$ y en la de Ernst Zermelo como $\left\{\left\{\left\{\varnothing_{\}}\right\}\right.\right.$. Benacerraf concluye de esto, primero, que los números no pueden reducirse a conjuntos $\mathrm{y}$, más importante, que ningún objeto, independientemente de sus propiedades, puede ser en sí mismo un número: "[L]o que es importante no es la individualidad de cada elemento sino la estructura que exhiben conjuntamente... Por lo tanto, sostengo, extendiendo el argumento que llevó a la conclusión de que los números no pueden ser conjuntos, que los números no pueden ser en absoluto objetos; pues no existe ninguna razón para identificar un número individual con un objeto particular que con otro [objeto] cualquiera"6.

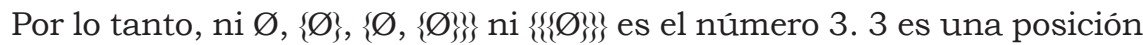
en una secuencia- $\omega$. Cualquier cosa puede jugar el rol de 3 en la medida en que sostenga relaciones estructurales adecuadas con los otros miembros de la secuencia- $\omega$ : "Cualquier objeto puede jugar el rol de 3; es decir, cualquier

\footnotetext{
5 Benacerraf, P., "What Numbers Could Not Be", en: Benacerraf, P. y H. Putnam (eds.) Philosophy of Mathematics. Selected Readings 2nd Edition, Cambridge: Cambridge University Press, 1983.

6 Ibid., pp. 290-291.
} 
objeto puede ser el tercer elemento en una progresión. Lo que es peculiar al 3 es que define ese rol -no al ser un paradigma de los objetos que pueden jugar dicho rol, sino al representar la relación que cualquier tercer miembro de una progresión conlleva con el resto de la progresión... La Aritmética es, por lo tanto, la ciencia que elabora la estructura abstracta que todas las progresiones tienen en común simplemente en virtud de ser progresiones"7.

El argumento de Benacerraf ha dado lugar a la postura llamada estructuralismo matemático, cuya idea principal es que las matemáticas son el estudio de patrones o estructuras. A pesar de que las observaciones de Benacerraf se hicieron, en parte, como una objeción al realismo matemático, el estructuralismo matemático no necesariamente se opone al realismo. De hecho, dos de los más importantes estructuralistas matemáticos, Stewart Shapiro y Michael Resnik, son realistas matemáticos. Shapiro define estructura como: "[L]a forma abstracta de un sistema, [obtenida] al resaltar las interrelaciones entre los objetos, e ignorando las características de los mismos que no afecten cómo se

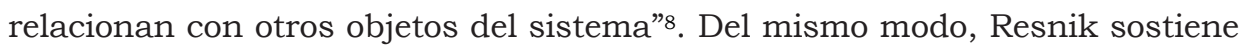
que: "[E]n matemáticas... no tenemos objetos con una composición 'interna' organizados en estructuras, solo tenemos estructuras. Los objetos de las matemáticas, esto es, las entidades que denotan nuestras constantes y cuantificadores, son puntos sin estructura o posiciones en estructuras. Como posiciones en estructuras, no tienen identidad o características fuera de la estructura"

Consideremos, por ejemplo, la estructura de un equipo de fútbol. Ella consiste en 11 posiciones relacionadas entre sí de manera específica. Estas posiciones son ocupadas por 11 jugadores. Los jugadores conforman un sistema físico que ejemplifica ${ }^{10}$ la estructura del equipo de fútbol $\mathrm{y}$, en ese sentido, los jugadores son jugadores de fútbol solo en la medida en que ocupan un lugar en la estructura de un equipo. Así, Paolo Guerrero es delantero solo en la medida en que ocupa la posición de delantero en un equipo de fútbol. Sin embargo, cualquier persona puede ocupar dicha posición, siempre y cuando esté en la relación correcta con el resto del equipo. Del mismo modo, cualquier objeto puede ser el tercer elemento en una progresión, siempre que guarde las

\footnotetext{
7 Ibid., p. 291. El énfasis es mío.

8 Shapiro, S., Thinking about Mathematics. The Philosophy of Mathematics, Oxford University Press, 2001, p. 259.

9 Resnik, M., "Mathematics as a Science of Patterns: Ontology and Reference", citado en: Shapiro, S., Thinking about Mathematics. The Philosophy of Mathematics, p. 259. Énfasis mío.

10 Traduzco la palabra instantiate como "ejemplificar" (a pesar de que en inglés también se usa exemplify en este contexto) para evitar el uso de "instanciar", que no está en el diccionario español.
} 
relaciones adecuadas con los otros miembros de la progresión. De dicho objeto se dice que ocupa la tercera posición en la estructura de los números naturales. Para el estructuralismo, por lo tanto, las matemáticas son el estudio de estas estructuras en cuanto tales.

\section{b) Estructuralismo ante rem}

Tanto Shapiro como Resnik sostienen que, a pesar de que los números no son en sí objetos sino posiciones en la estructura de los números naturales, sí tiene sentido hablar en términos de objetos matemáticos, ya que nos podemos referir a las posiciones de estructuras como objetos. Por ejemplo, podemos hablar de aspectos estructurales de los equipos de fútbol sin referirnos a ningún equipo en particular o a sus miembros, como cuando decimos que "el arquero es el único jugador que puede usar las manos". En esos casos, no nos referimos a ningún arquero en particular, sino a la posición de arquero en tanto objeto. Lo mismo puede decirse de las posiciones en las estructuras matemáticas. La posición 3 confiere la propiedad de ser "primo" (de manera relativa a los otros miembros de la progresión) al objeto que ocupa el tercer lugar. En ese sentido, en palabras de Shapiro, "las posiciones en las estructuras matemáticas son tan objetos como cualquier otro"11.

Shapiro y Resnik son estructuralistas ante rem. Para ellos, las estructuras matemáticas son ontológicamente anteriores a los sistemas que las ejemplifican. Estas estructuras matemáticas existen independientemente de si son ejemplificadas o no. De hecho, para ambos autores, algunas estructuras matemáticas (tales como los números transfinitos de Cantor) existen a pesar de que no pueden ejemplificarse en nuestro mundo físico. En ese sentido, Shapiro y Resnik son realistas matemáticos. Ellos creen en la existencia objetiva de objetos matemáticos abstractos, pero, al mismo tiempo, son estructuralistas matemáticos, pues sostienen que estos objetos son posiciones en estructuras matemáticas.

Algunos autores han criticado el estructuralismo ante rem señalando que, ya que finalmente sostiene que existen objetos en un espacio no físico y platónico, no habria mucha diferencia entre el estructuralismo y el platonismo matemático puro. Ian Hacking, por ejemplo, llama al estructuralismo ante rem "platonismo disfrazado"12. Sin embargo, una diferencia entre estas dos

\footnotetext{
${ }^{11}$ Shapiro, S., Philosophy of Mathematics. Structure and Ontology, Nueva York: Oxford University Press, 1997, p. 89.

${ }^{12}$ Hacking, I., Why Is There Philosophy of Mathematics At All?, Cambridge: Cambridge University Press, 2014, p. 237.
} 
posturas es que los objetos de los estructuralistas ante rem son posiciones en una estructura. Estas posiciones pueden ser ocupadas por otros objetos, incluyendo objetos físicos concretos. Así es como el estructuralismo ante rem da cuenta de la relación entre el mundo matemático abstracto y el mundo físico concreto, confiriendo así lo que W.O. Quine llamaba "el carácter empírico" de las matemáticas. Los sistemas físicos ejemplifican estructuras matemáticas y obtienen las propiedades de estas estructuras. En principio, podemos aprender acerca de las estructuras matemáticas analizando sus ejemplificaciones fisicas. Esta opción, sin embargo, no está abierta a los platonistas matemáticos puros.

Un reto del estructuralismo ante rem es justificar la existencia de estructuras matemáticas no ejemplificadas o no ejemplificables. ¿Cómo podemos estar seguros de que estas estructuras no ejemplificadas no son un simple producto de nuestra imaginación? Responder a esta pregunta es crucial para la plausibilidad de esta postura pues, si no se puede justificar la existencia de las estructuras no ejemplificadas (por ejemplo, si las estructuras no ejemplificadas fueran una simple ficción), no tendría sentido interpretar a las estructuras fisicas como ejemplificaciones de estructuras matemáticas, porque las categorias ficticias no tienen ejemplificaciones reales: nadie es ni puede ser Bilbo, aunque camine descalzo, tenga un anillo y fume tabaco en pipa. De la misma forma, a menos que se establezca la existencia de estructuras matemáticas, no tiene sentido llamar a los dedos de mi mano una ejemplificación de secuencia de la estructura de los números naturales. En la sección 3, veremos con detalle este problema y explicaremos la importancia de la solución que alude a la aplicabilidad de las matemáticas en ciencia ${ }^{13}$.

\subsection{Ficcionalismo}

Tal vez la versión más extrema de antirrealismo sea el ficcionalismo matemático. Esta postura sostiene que las matemáticas son una historia sobre entidades ficticias. De acuerdo al ficcionalismo, ya que cuantifican sobre objetos ficcionales, las afirmaciones matemáticas pueden ser interpretadas literalmente: la mayoría son simplemente falsas. La afirmación "existen infinitos números primos" es falsa porque no existen los números primos. Sin embargo, hay un sentido en el que dicha afirmación es verdadera: dentro de la historia (es decir, del cuento) de las matemáticas, la afirmación es verdadera, tal como las afirma-

\footnotetext{
${ }^{13}$ Para facilitar esta presentación, voy a ignorar el estructuralismo in re de Geoffrey Hellman (Hemlan, G., Mathematics Without Numbers: Towards a Modal-Structural Interpretation, Oxford: Oxford University Press, 1989).
} 
ciones sobre historias ficticias son verdaderas: "Frodo es el sobrino de Bilbo", "Rohan está al sureste de La Comarca", etcétera, son todas verdaderas dentro del universo de El Señor de los Anillos, pero no son verdaderas absolutamente. Tal como lo pone Hartry Field: "[E]1 ficcionalista cree que $2+2=4$ solamente en el sentido de que él o ella cree que las matemáticas estándar dicen (o tienen como consecuencia) que $2+2=4 " 14$.

Otras afirmaciones son trivialmente verdaderas, como por ejemplo "no existe un número primo que sea el mayor de todos". Esta afirmación es verdadera simplemente porque no existen los números primos.

Algunos sostienen que el ficcionalismo matemático es implausible porque las matemáticas tienen su origen en nuestras percepciones de la realidad y, en ese sentido, tienen una especie de anclaje en el mundo físico. Por esa razón, continúa el argumento, las matemáticas no pueden ser completamente ficcionales. Sin embargo, yo no creo que este sea un problema. Las ficciones (por ejemplo, las ficciones literarias) frecuentemente tienen, también, un anclaje en la realidad, pero luego se alejan de sus orígenes reales y postulan entidades y procesos ficticios. Según el ficcionalismo, esto es exactamente lo que sucede con las matemáticas.

Otra objeción a aquella postura se basa en el requerimiento de Paul Benacerraf de que debemos manejar una "semántica uniforme", es decir, debemos tener "una teoría semántica homogénea en la que las proposiciones matemáticas sean paralelas a la semántica del resto del lenguaje"15. La objeción es que ni los matemáticos ni la gente común suele hablar en términos de "la historia de las matemáticas". Dicen, simplemente, cosas como "existen infinitos números primos", sin la calificación de acuerdo a la aritmética. En ese sentido, el ficcionalismo parece requerir una reinterpretación de la práctica matemática y cotidiana, lo cual iría contra el requisito de la semántica uniforme.

Sin embargo, yo no creo que esta objeción se justifique. De hecho, esta objeción se basa en una interpretación errónea de la práctica matemática. En la práctica, las afirmaciones matemáticas siempre se hacen asumiendo ciertas cosas sobre el dominio de aplicación. En otras palabras, los matemáticos nunca toman las afirmaciones matemáticas directamente, es decir, sin calificaciones. Esta es una diferencia fundamental entre el discurso matemático y el discurso científico: la ciencia lidia con una realidad única; las matemáticas,

\footnotetext{
${ }^{14}$ Field, H., Realism, Mathematics \& Modality, Oxford: Basil Blackwell, 1989, p. 3.

${ }^{15}$ Benacerraf, P., "Mathematical Truth", en: Benacerraf, P. y H. Putnam (eds.), Philosophy of Mathematics. Selected Readings, Cambridge: Cambridge University Press, 1983, p. 403.
} 
con un espacio matemático definido por los axiomas específicos a una rama matemática ${ }^{16}$. Esta idea ha sido enfatizada por Otávio Bueno: "El ficcionalista no introduce un operador ficcional a las afirmaciones matemáticas. Las afirmaciones se usan en el contexto de principios que caracterizan las propiedades de los objetos matemáticos relevantes. En ese sentido, el operador ficcional -en el sentido de principios comprehensivos que especifican un cierto dominio de objetos- está ya puesto como parte de la práctica matemática. El ficcionalista no está añadiendo un nuevo elemento al lenguaje matemático. Bajo una conceptualización adecuada, el operador ficcional ya está ahỉ”17.

El siguiente ejemplo puede ayudar a comprender la idea de Bueno. Supongamos que se nos pregunta si la afirmación "la suma de los ángulos internos de un triángulo es $180^{\circ}$ " es verdadera. Nos podemos sentir inclinados a decir "sí lo es", pero, evidentemente, una respuesta más apropiada sería decir "depende". ¿Por qué? Porque, si estamos hablando de triángulos en un espacio euclidiano, la respuesta sería "sí", pero si estamos hablando de triángulos en un espacio no euclidiano, la respuesta sería "no", y podríamos hacer referencia a la geometría de Riemann, donde la suma de los ángulos internos de un triángulo es mayor a $180^{\circ}$. El punto es que la práctica matemática introduce de antemano dicha calificación a las afirmaciones matemáticas. Por lo tanto, el ficcionalista no requiere un cambio en las prácticas comunes de hacer matemáticas.

\section{Importancia de la aplicabilidad de las matemáticas en el debate sobre el realismo matemático}

\subsection{El problema del acceso epistémico}

Tal como mencioné, para los realistas, los objetos matemáticos son abstractos, acausales y no están localizados en el espacio-tiempo. Sin embargo, esta postura enfrenta el siguiente problema: ¿Cómo puede ser que los humanos

\footnotetext{
${ }^{16}$ Por supuesto, alguien podría decir que en realidad todas nuestras verdades son relativas a una historia contextual y que, en sentido estricto, no hay diferencia entre el discurso ficticio de las historias de J.R.R Tolkien y nuestras afirmaciones cotidianas acerca de mesas y manzanas: las matemáticas serian una ficción porque todo es una ficción. Este argumento en favor del ficcionalismo matemático se basa en un antirrealismo generalizado incluso más fuerte que el antirrealismo científico (que propone ya sea agnosticismo o rechazo de plano de las entidades inobservables postuladas por la ciencia). La razón por la que voy a ignorer esta postura es que va en contra de la estrategia que he adoptado en este artículo, que consiste en criticar el realismo matemático mínimamente. Mi objetivo es mostrar que incluso si uno fuera realista científico, no tendría razones para ser realista matemático.

17 Bueno, O., "Mathematical Fictionalism", en: Bueno, O. y O. Linnebo (eds.), New Waves in Philosophy of Mathematics, Nueva York: Palgrave Macmillan, 2009, p. 76.
} 
hayamos adquirido conocimiento sobre estas entidades abstractas y acausales? A esto se le llama el problema del acceso epistémico, también conocido como "el reto de Benacerraf", y consiste en hacer que nuestra "explicación de las verdades matemáticas coincida con una epistemología razonable"18. El reto es, justamente, brindar una explicación de cómo nosotros, seres concretos, causalmente afectados por nuestro entorno, tenemos conocimiento de estos objetos acausales.

Una solución bastante conocida a este problema es la de Kurt Gödel. De acuerdo a Gödel: "[Los humanos tenemos] algo como una percepción... de los objetos de la teoría de conjuntos, tal como se evidencia en el hecho de que los axiomas se imponen sobre nosotros como verdaderos. No veo ninguna razón por la que deberiamos confiar menos en este tipo de percepción, es decir, en la intuición matemática, que en la percepción sensorial"19.

La respuesta de Gödel consiste en que podemos conocer los objetos matemáticos justamente porque tenemos una manera de percibirlos. Sin embargo, aunque no completamente circular, esta respuesta es ad hoc, pues el problema surge precisamente por la falta de explicación de dicha facultad de percepción matemática. El problema es la aparente incompatibilidad de esta percepción matemática con una visión naturalista del mundo ${ }^{20}$. Shapiro resume la preocupación en estos términos: “¿Cómo es posible que los humanos, organismos físicos habitando un universo físico, tengamos un conocimiento intuitivo de este reino de objetos abstractos causalmente inertes? ¿Cómo es que la mente humana, tal como la describe la psicología empírica, pueda conocer algo sobre conjuntos y números tal como se describen en matemáticas?”21.

\subsection{Respuestas al problema del acceso epistémico}

En el ámbito contemporáneo, las respuestas a este problema varían. James Cargile, por ejemplo, sostiene que esta objeción comete una petición de principio $^{22}$. Si los objetos matemáticos no están localizados en el espacio-tiempo, entonces están excluidos, por definición, de una visión naturalista del mundo. Por lo tanto, no sería justo rechazar su existencia simplemente porque no se

\footnotetext{
18 Benacerraf, P., "Mathematical Truth", p. 403.

19 Gödel, K., "What is Cantor's Continuum Problema?", en: Benacerraf, P. y H. Putnam (eds.), Philosophy of Mathematics. Selected Readings 2nd Edition, Cambridge: Cambridge University Press, 1983, pp. 483-484.

${ }^{20}$ Por una descripción más detallada del naturalismo, ver la sección 4.3.

21 Shapiro S., Thinking about Mathematics. The Philosophy of Mathematics, p. 221.

22 Cargile, J., “On 'Alexander's' Dictum”, en: Topoi, v. XXII, 2 (2003), pp. 143-149, p. 144.
} 
ajustan a nuestras creencias naturalistas. Después de todo, uno siempre puede tomar el fracaso en dar cuenta del conocimiento matemático como argumento para rechazar el naturalismo.

Otra respuesta consiste en distinguir entre el contexto en el que uno descubre afirmaciones matemáticas y el contexto en el que uno justifica dichas afirmaciones, y sostener que, si tenemos una buena justificación de estas afirmaciones, no necesitamos brindar una explicación de cómo así llegamos a tomar conocimiento de ellas. La idea es que es un hecho que poseemos un sistema de afirmaciones matemáticas $\mathrm{y}$, si mostramos que estamos justificados, de alguna manera, en interpretar estas afirmaciones literalmente, habriamos mostrado que los objetos matemáticos existen. Esto sería verdad independientemente de los medios a través de los cuales habríamos tomado conocimiento de dichas afirmaciones. La idea principal de esta respuesta es que descubrir y justificar afirmaciones matemáticas son dos actividades distintas y solo la última es relevante para resolver la pregunta sobre el realismo matemático. Hecha esta distinción, los realistas matemáticos pueden brindar diferentes justificaciones, aunque, como hemos visto, no todas ellas satisfarian a filósofos naturalistas.

De la discusión precedente podemos concluir que el problema del acceso epistémico no es determinante contra la postura realista. Intentemos entonces plantear la discusión de manera más precisa. ¿Cómo podemos distinguir entre afirmaciones que cuantifican sobre objetos reales y afirmaciones que cuantifican sobre ficciones? ¿Debemos poner a los números primos junto a las esmeraldas o junto a Bilbo? Como veremos en la sección siguiente, muchos realistas matemáticos apelan a la aplicabilidad de las matemáticas en ciencia para justificar su postura.

\section{La aplicabilidad de las matemáticas en ciencia}

En la filosofia de las matemáticas contemporánea existen diferentes justificaciones del realismo matemático, incluyendo la defensa de Cargile ${ }^{23} \mathrm{del}$ platonismo matemático puro, o Shapiro ${ }^{24} \mathrm{y}$ su justificación coherentista del estructuralismo ante rem. Sin embargo, una de las justificaciones más discutidas actualmente se basa en la aplicabilidad de las matemáticas en ciencia ${ }^{25}$. La idea

\footnotetext{
${ }^{23}$ Cargile, J., "On 'Alexander's' Dictum”, 2003.

${ }^{24}$ Shapiro, S., Philosophy of Mathematics. Structure and Ontology, Nueva York: Oxford University Press, 1997.

${ }^{25}$ Por ejemplo, Baker, A., "Are there Genuine Mathematical Explanations of Physical Phenomena?", en: Mind, v. CXIV (2005), pp. 223-238; "Mathematical Explanation in Science", en: British Journal
} 
es que, si las matemáticas fueran una simple ficción, no serían compatibles con una comprensión científica del mundo. Esta justificación ha adoptado diferentes modalidades. Primero, uno podría preguntarse por qué las matemáticas serían tan útiles en ciencia si fueran una simple ficción (el problema de la utilidad). Adicionalmente, uno podría preguntarse por qué es frecuente el caso de que conceptos desarrollados con un propósito puramente matemático terminan, luego, siendo útiles en aplicaciones científicas, o por qué se puede hacer, a veces, predicciones físicas analizando simplemente las formulaciones matemáticas de las leyes de la naturaleza (el problema de los usos no esperados). Finalmente, uno podría preguntarse por qué las matemáticas parecen ser indispensables para nuestras mejores teorias cientificas, en particular, en nuestras mejores explicaciones científicas. En todos estos casos, la idea es que la única manera de explicar estas aplicaciones exitosas es adoptando una postura realista -en particular, una postura que dé cuenta del aspecto "empírico" de las matemáticas, en el sentido de explicar la relación entre las estructuras matemáticas y los sistemas físicos, tal como lo hace el estructuralismo ante rem con la noción de ejemplificación-. En esta sección voy a discutir y refutar cinco argumentos realistas basados en la (mera) utilidad de las matemáticas en ciencia (4.1), las aplicaciones inesperadas o "sorpresivas" (4.2) y el problema de la indispensabilidad (4.3-4.5).

\subsection{Argumento 1: La mera utilidad de las matemáticas en ciencia}

Para los ficcionalistas, al igual que El Señor de los Anillos de J.R.R. Tolkien, las matemáticas son una historia sobre entidades ficticias. El problema, por supuesto, es que las historias de Tolkien tienen un rango limitado de aplicación en ciencia. Uno podría utilizarlas para aprender algo sobre el mundo como, por ejemplo, el valor de la hospitalidad, pero de ninguna manera pueden ser usadas como la herramienta científica por excelencia. Ese lugar está reservado para las matemáticas. La razón, se ha dicho frecuentemente, es que las matemáticas lidian con la estructura subyacente del mundo. Una versión de este argumento puede reconstruirse de la siguiente manera: 
P1) Casi todas las disciplinas científicas usan matemáticas.

P2) La única razón por la que casi todas las disciplinas científicas usan matemáticas es que los objetos matemáticos existen.

C) Los objetos matemáticos existen.

\section{Respuestas}

La primera premisa es evidente. Prácticamente basta leer cualquier artículo científico al azar. La segunda premisa es más problemática pues, como veremos, hay otras razones que explican la destacada utilidad de las matemáticas.

Antes de continuar, voy a mencionar una mala explicación de la utilidad de las matemáticas en ciencia: uno podría decir que las matemáticas son una creación humana, inspirada en eventos reales del mundo (por ejemplo, nuestra experiencia perceptiva de patrones y regularidades), y que, por lo tanto, no es en absoluto sorprendente que estos objetos sean luego utilizados en ciencia: la ciencia lidia con el mundo; los objetos matemáticos han sido creados a partir del mundo; por lo tanto, no es problemático que las ciencias usen matemáticas. Esta no es una buena explicación de la utilidad de las matemáticas. El hecho en sí de que las matemáticas se hayan creado tomando como base el mundo real no explica por qué son tan útiles en la ciencia. Muchas ficciones, tales como, por ejemplo, las ficciones literarias, también se inspiran frecuentemente en hechos reales y, sin embargo, no son tan útiles en ciencia como las matemáticas. Las historias de Harry Potter están ciertamente inspiradas en el sistema escolar británico, pero muy pocas explicaciones científicas usan esas historias para comprender dicho sistema.

Una respuesta más interesante surge cuando prestamos atención al proceso mismo de aplicación de las matemáticas y vemos que el uso científico de las matemáticas requiere que el fenómeno estudiado sea "matematizado" primero. En un sentido, esto explicaría por qué las matemáticas son tan ampliamente utilizadas. Tal como ha señalado Patrick Suppes, el proceso de aplicación de las matemáticas al mundo no se da de manera directa, sino a través de una serie de modelos y pasos, organizados jerárquicamente, que median entre los modelos teóricos y el sistema examinado ${ }^{26}$. El punto básico aquí es que, para que un fenómeno físico pueda ser estudiado matemáticamente, primero debe ser medido. Este proceso de medición implica, en sí mismo, una matematización,

${ }^{26}$ Suppes, P., "Models of Data", en: Nagel, E. y otros (eds.), Logic, Methodology, and Philosophy of Science: Proceedings of the 1960 International Congress, Stanford: Stanford University Press, 1962. 
esto es, asignar afirmaciones mixtas que involucran elementos fisicos y matemáticos (por ejemplo, "tres manzanas"), que luego son representados como conjuntos de datos. Estos conjuntos de datos son la base de una jerarquía de modelos que concluyen con modelos teóricos. El punto es que, para describir esta versión matematizada de la realidad, no es en absoluto sorprendente que tengamos que usar afirmaciones matemáticas o que algunos conceptos matemáticos sean útiles.

\subsection{Argumento 2: Los usos inesperados de las matemáticas}

Tal como señala el premio Nobel en fisica Eugene Wigner, es frecuente que conceptos matemáticos desarrollados por propósios puramente estéticos o formales terminen siendo útiles en la ciencia. Esto es lo que él llama La irrazonable efectividad de las matemáticas (1960). Un ejemplo típico son los números complejos. Jerome Cardan, quien formuló la teoría de los números complejos en el siglo XVI, nunca pretendió que estos números sean en absoluto útiles. Más bien, señaló lo siguiente acerca de su propia creación: "Así progresa sutilmente la aritmética, cuyo fin, como se dice, es tan refinado como inútil" 27 . Sin embargo, señala Wigner, los números complejos son actualmente de gran importancia en nuestras mejores teorías físicas: "[L]os números complejos están lejos de ser naturales o simples y no son sugeridos por observaciones físicas... [Sin embargo] el uso de los números complejos... no es un truco para hacer cálculos en matemática aplicada sino que han llegado a ser una necesidad en la formulación de las leyes de la mecánica cuántica"28.

Según Wigner, esta "irrazonable efectividad" de las matemáticas es "algo bordeando lo misterioso", sobre lo cual "no existe una explicación racional" 29 . La idea de Wigner es que, a pesar de la diferencia entre las metodologías en matemática y en ciencia -a saber, que la ciencia se lleva por evidencia empírica, mientras que las matemáticas parecen avanzar, en mayor medida, desconectadas del mundo-, las matemáticas son fundamentales en ciencia.

Otro tipo de aplicaciones inesperadas se da en aquellos casos en los que el mero análisis de las ecuaciones matemáticas que describen las leyes naturales lleva a realizar predicciones que son la fuente de descubrimientos científicos posteriores. En un famoso pasaje, el fisico Heinrich Hertz explica

\footnotetext{
${ }^{27}$ Citado en Bangu, S., "On The Unreasonable Effectiveness of Mathematics in the Natural Sciences", en: Ippoliti, E. y otros, Models and Inferences in Science, Springer, 2016.

${ }^{28}$ Wigner, E., "The Unreasonable Effectiveness of Mathematics in the Natural Sciences", en: Communications on Pure and Applied Mathematics, v. XIII (1960), pp. 1-14, p. 5.

${ }^{29}$ Ibid., p. 1.
} 
este fenómeno de la siguiente manera: "Uno no puede evadir la sensación de que estas fórmulas matemáticas tienen una existencia independiente y una inteligencia en sí mismas, de que son más sabias que nosotros, más sabias incluso que aquellos quienes las descubrieron, que sacamos más de ellas de lo que inicialmente pusimos"30.

Un caso que se suele citar para ilustrar este punto es el descubrimiento del positrón por Paul Dirac. Simplemente analizando el formalismo matemático, Dirac encontró soluciones negativas a sus ecuaciones. Sin embargo, en vez de rechazarlas como meros artefactos de las matemáticas, postuló la existencia de partículas de igual masa que los electrones, pero con carga opuesta. Esto condujo a la idea del positrón, partícula que fue luego descubierta experimentalmente. Otro caso famoso es el de las transformaciones de Edward Lorentz, desarrolladas con fines puramente matemáticos y que luego fueron interpretadas en términos del espacio-tiempo de la teoría de la relatividad por Albert Einstein $^{31}$. La idea sería que las matemáticas "saben más" acerca del mundo que nosotros.

Ahora bien, ni Wigner ni Hertz extrajeron explícitamente la conclusión de que estas aplicaciones inesperadas justificaban el realismo matemático. De hecho, hasta donde yo sé, estas aplicaciones inesperadas no han sido utilizadas por filósofos para justificar esta posición. Hay, sin embargo, algunos autores de literatura científica de divulgación que sí lo han hecho. El físico Roger Penrose, por ejemplo, toma estos usos inesperados como evidencia de un "acuerdo entre matemáticas y física... entre el mundo de Platón y el mundo físico"32. La razón subyacente, sostiene Penrose, es que estos dos mundos "son el mismo"33. Desafortunadamente, Penrose no especifica a qué se refiere con "el mismo", pero, como hemos visto, una posible explicación sería que Penrose suscribe una versión de estructuralismo ante rem. Es decir, la estructura subyacente del mundo físico es matemática porque el mundo físico ejemplifica estructuras matemáticas. De manera similar, el físico Paul Davies sostiene que la razón por la que matemáticas desarrolladas como un ejercicio abstracto son "espectacularmente efectivas en las teorías físicas" es que las matemáticas "descubren

\footnotetext{
${ }^{30}$ Citado en Colyvan, M., An Introduction to the Philosophy of Mathematics, Cambridge: Cambridge University Press, 2012, p. 99.

${ }^{31}$ Estos ejemplos se discuten en Colyvan, M., The Indispensability of Mathematics, Oxford: Oxford University Press, 2001, pp. 81-85.

${ }^{32}$ Penrose, R., The Eperor's New Mind: Concerning Computers, Minds, and the Laws of Physics, Oxford: Oxford University Press, 1989, p. 430.

${ }^{33}$ Ibid.
} 
alguna propiedad real de la naturaleza"34, a saber, que el mundo matemático y el mundo físico son estructuralmente el mismo.

\section{Respuestas}

A pesar de Penrose y Davies, el realismo matemático no es la única explicación de estas aplicaciones inesperadas. Por ejemplo, Paul Humphreys señala que la "sorpresa" de estas aplicaciones desaparece si prestamos atención a la vastedad del ámbito matemático: "Debido a que el ámbito de estructuras matemáticas es mucho más grande que el ámbito de estructuras físicas idealizadas, no es en absoluto sorprendente que uno pueda encontrar, o construir, objetos en el primer ámbito que se apliquen al segundo"35.

La idea es que, ya que las matemáticas son una inmensa fuente de estructuras, es razonable pensar que algunas de estas estructuras van a ser útiles en comprender y representar estructuras físicas ${ }^{36}$. Esto es incluso más evidente cuando consideramos que las matemáticas no describen o representan directamente estructuras fisicas, sino versiones idealizadas de estas estructuras fisicas. Adicionalmente, es frecuente el caso de que una misma estructura matemática pueda emplearse para describir aspectos comunes a diferentes estructuras físicas. Humphreys señala, por ejemplo, que es posible representar, con el mismo modelo matemático, a saber, el modelo de Poisson, fenómenos tan variados como organismos por unidad de volumen en una solución diluida, llamadas telefónicas, autos, decaimiento radioactivo, cromosomas, bombas voladoras, peces capturados, etcétera ${ }^{37}$.

La disponibilidad de estructuras matemáticas para representar fenómenos físicos se hace más evidente si tomamos en cuenta que, en cierto sentido, existe un sesgo en el proceso de selección: los científicos trabajan con estructuras fisicas que son adaptables al proceso de matematización y luego "fuerzan" sus modelos dentro de las matemáticas disponibles ${ }^{38}$. Es decir, los científicos no solo describen, primero, el mundo de manera tal que es representable matemáticamente (tal como vimos en 4.1), sino que además tienden a enfocarse en aquellos fenómenos que se adaptan a este patrón. Por estas razones, sostiene Humphreys, no es en absoluto sorprendente que algunas estructuras matemá-

\footnotetext{
34 Davies, P., The Mind of God, Londres: Penguin Book, 1992, p. 151.

${ }^{35}$ Humphreys, P., Extending Ourselves, Oxford: Oxford University Press, 2004, p. 90.

${ }^{36}$ Algo que puede hacernos ver la vastedad del ámbito matemático es que el número $10^{83}$ es bastante más grande que el número de átomos en el universo observable.

37 Ver detalles en Humphreys, P., o.c., pp. 88-91.

38 Ibid., p. 90.
} 
ticas sean útiles de maneras nuevas e inesperadas. Simplemente sucede que, a veces, descubrimos que los sistemas fisicos y las estructuras matemáticas que utilizamos para representarlos tienen más en común de lo que habíamos supuesto inicialmente. En los casos opuestos, es decir, cuando tenemos fenómenos físicos que presentan aspectos no contemplados en la representación matemática inicialmente asignada, sencillamente cambiamos de representación matemática y usamos otra de las tantas disponibles.

Ahora bien, sería un error interpretar las observaciones de Humphreys como si el proceso de aplicación consistiera, simplemente, en encontrar una estructura matemática que encaje en el fenómeno en cuestión (actitud que Otávio Bueno y Stephen French llaman "optimismo matemático perezoso"), o el mero proceso de esperar a ver si existen estructuras fisicas capaces de ser descritas matemáticamente (Bueno y French llaman a esta actitud "oportunismo matemático"39). El punto de Humphreys es que, ya que las estructuras físicas tienen, primero, que ser idealizadas para que los modelos matemáticos sean útiles, el hecho de que haya estructuras matemáticas adecuadas que puedan ser adoptadas por esos modelos no es sorprendente.

Otro aspecto relevante del proceso de aplicación de las matemáticas, señalado por Otávio Bueno, tiene que ver con el hecho de que los científicos nunca "leen" hechos fisicos directamente a partir de hechos matemáticos, sino que siempre tienen que interpretar físicamente los resultados obtenidos en sus modelos. Una misma estructura matemática puede ser interpretada de diferentes maneras y, en ese sentido, el simple análisis de la estructura matemática no es suficiente para determinar qué interpretación es la correcta ${ }^{40}$. En palabras de Bueno: "[L]as expresiones matemáticas, tomadas en sí mismas, no son sobre eventos fisicos en el mundo: tienen que ser, en primer lugar, (propiamente) interpretadas antes de que sean relevantes para la descripción de los fenómenos fisicos. Una ecuación diferencial tiene múltiples interpretaciones, y dependiendo de la interpretación que uno adopte, la ecuación puede no tener en absoluto implicaciones respecto del mundo fisico, o puede tener implicaciones que resulten ser empíricamente inadecuadas, o implicaciones que, adecuadamente reconstruidas, capturen algunos aspectos del mundo fisico" ${ }^{41}$.

\footnotetext{
${ }^{39}$ Bueno, O. y S. French, The Applicability of Mathematics in Science: Immersion, Inference and Interpretation, Oxford: Oxford University Press, 2018.

${ }^{40}$ Bueno, O., "An Easy Road to Nominalism", en: Mind, v. CXXI (2012), p. 980.

${ }^{41}$ Ibid., p. 973.
} 
El punto de Bueno es que, sin una interpretación, "las matemáticas no dicen nada acerca del mundo físico" 42 . Esto se puede ver si se examina con atención el caso del descubrimiento de los positrones. Dirac no leyó una interpretación en términos de positrones directamente de sus ecuaciones. De hecho, primero pensó que estas soluciones negativas eran simples artefactos de las matemáticas sin implicaciones fisicas; luego, en un segundo momento, las interpretó como refiriéndose a "agujeros" en el espacio-tiempo. Esta segunda interpretación, sin embargo, es inconsistente con su teoría, pues implica la existencia de partículas de masa infinita. Finalmente, Dirac interpretó estas soluciones negativas como refiriéndose a partículas de la misma masa que los electrones, pero con carga opuesta, dando así lugar a la idea del positrón. Hasta este momento, la interpretación en términos de positrones se sigue tomando como correcta ${ }^{43}$. Nuevamente, el punto es que las soluciones de la ecuación de Dirac carecen de significado en sí mismas, pues se les pueden asignar diferentes interpretaciones. Es solo cuando se les asigna una interpretación correcta que el formalismo matemático captura aspectos del mundo ${ }^{44}$.

La idea principal aquí es que las matemáticas no nos brindan una descripción directa del mundo. Las matemáticas no lidian con el mundo. Es solo cuando los resultados matemáticos se interpretan en términos empíricos que se vuelven útiles en ciencia. Por esa razón, decir simplemente que las matemáticas son útiles no es un argumento en favor del realismo matemático.

\subsection{Argumento 3: La indispensabilidad de las matemáticas en la ciencia}

El caso anterior en favor del realismo matemático se basa en la mera utilidad de las matemáticas en la ciencia. Sin embargo, es posible construir un argumento más fuerte si nos enfocamos no solo en el hecho de que las matemáticas son útiles en ciencia, sino en que son (o parecen ser) indispensables para hacer ciencia. Este ha sido llamado el argumento de indispensabilidad (AI) y varios autores de ambos lados del debate como, por ejemplo, el realista matemático Mark Colyvan y el ficcionalista Hartry Field, concuerdan en que este es, tal vez, el argumento más importante para justificar el realismo matemático ${ }^{45}$. La idea es que, si uno considera razonable el realismo científico, que es la creencia en la existencia de los objetos y procesos inobservables postu-

\footnotetext{
42 Ibid.

43 Ibid., p. 974.

44 Cf., Bueno, O., "An Easy Road to Nominalism", y Bueno, O. y M. Colyvan, "An Inferential Conception of the Application of Mathematics", en: Noûs, v. XLV, 2 (2011), pp. 345-374.

45 Menciono algunas excepciones en la conclusión.
} 
lados por las mejores formulaciones de nuestras mejores teorías científicas (por ejemplo, electrones, quarks, genes, campos electromagnéticos, procesos evolutivos, etcétera), también debería considerar razonable sostener el realismo matemático, es decir, la creencia en la existencia de los objetos abstractos a los que se refieren las afirmaciones matemáticas que aparecen en las teorias científicas. Por supuesto, no todos los filósofos son realistas científicos, pero, tal como señala Hilary Putnam, el punto del AI es que, si uno admite que las entidades inobservables concretas existen, entonces sería intelectualmente deshonesto si no admitiera que los objetos matemáticos existen también ${ }^{46}$.

En esta subsección voy a analizar la primera versión del AI, basada en el naturalismo y el holismo confirmacional de W.O. Quine. De acuerdo a este filósofo, el naturalismo es: "[E]1 abandono del objetivo de una primera filosofia. Ve a la ciencia natural como una indagación en la realidad, falible y corregible, pero que no responde a ningún tribunal supra-científico, y que no requiere de ninguna justificación más allá de la observación y el método hipotético-deductivo"47. Del mismo modo, Quine defendia la tesis del holismo confirmacional, que consiste en que las afirmaciones científicas no se confirman o refutan aisladamente, sino como parte de un sistema de hipótesis. En otras palabras, las hipótesis científicas nunca se ponen a prueba de manera aislada, sino en conjunto con hipótesis auxiliares: "La totalidad de nuestro llamado conocimiento, o creencias, desde los aspectos más casuales de geografía e historia hasta las leyes más profundas de la física atómica o incluso de las matemáticas puras y lógica, es un entramado construido por los humanos que se conecta con la experiencia solamente en los bordes"48.

De acuerdo a esta postura, si la evidencia empírica confirma parte de una teoría, toda la teoría queda confirmada, incluyendo sus componentes matemáticos. Ya que las matemáticas cuantifican sobre números, funciones, conjuntos, etcétera, la creencia en la existencia de dichas entidades queda justificada. En palabras de Quine: "[E]1 discurso científico está tan irremediablemente comprometido con objetos abstractos -naciones, especies, números, funciones, conjuntos- como lo está con manzanas y otros cuerpos. Todas estas cosas figuran como valores de las variables de nuestro sistema total del mundo.

\footnotetext{
${ }^{46}$ Colyvan, M., The Indispensability of Mathematics, pp. 10-11.

${ }^{47}$ Citado en Resnik M., "Quine and the Web of Belief", en: Shapiro, S. (ed.), The Oxford Handbook of Philosophy of Mathematics and Logic, Oxford: Oxford University Press, 2005, p. 416.

${ }^{48}$ Citado en ibid., p. 414. El énfasis es mío.
} 
Los números y las funciones contribuyen tan genuinamente a las teorias fisicas como las partículas hipotéticas ${ }^{49}{ }^{\prime 50}$.

Mark Colyvan reconstruye el AI de la siguiente manera:

P1) Tenemos que aceptar la existencia de los objetos que son indispensbles para el éxito de nuestras mejores teorías científicas.

P2) Cuantificar sobre objetos matemáticos es indispensable para el éxito de nuestras mejores teorias científicas.

C) Debemos aceptar la existencia de objetos matemáticos ${ }^{51}$.

La idea del AI es que, debido al naturalismo, tenemos razones para considerar verdaderas las afirmaciones de nuestras mejores teorías científicas y, debido al holismo, debemos aceptar todos los objetos y procesos sobre los que cuantifican dichas afirmaciones. Ya que cuantificar sobre objetos matemáticos es indispensable para el éxito de dichas teorías, tenemos que aceptar también la existencia de los objetos matemáticos mencionados en dichas afirmaciones.

\section{Respuesta}

Penelope Maddy critica el AI basándose en que, de acuerdo a ella, existe una incompatibilidad entre el naturalismo y el holismo confirmacional. Para Maddy, no es cierto que debamos aceptar todas las entidades que son indispensables para nuestras mejores teorias científicas ${ }^{52}$. Ella no rechaza el postulado naturalista de que solo la ciencia puede decirnos lo que existe y es verdadero. Su punto es que no todas las entidades indispensables en ciencia existen. Es importante notar que su crítica se aplica a las teorías científicas en general y no solo en su relación con teorías matemáticas, es decir, Maddy apunta a problemas reconciliando el naturalismo con el holismo confirmacional, independientemente del AI. Consideremos los siguientes ejemplos: en dinámica de fluidos, se asume que la materia es continua, pero los científicos no consideran que la materia sea continua; al explicar las olas del mar, se asume que el océano es infinitamente profundo, pero nadie realmente cree que este sea el caso; en mecánica clásica, algunas propiedades cinemáticas se calculan asumiendo planos sin fricción, pero dichos planos no existen en el mundo. Como señala Maddy: "Si podemos ver, en el curso de nuestro estudio científico de la ciencia, que ciertas partes

\footnotetext{
49 Partículas hipotéticas, o teóricas, son las que yo he llamado arriba "inobservables".

${ }^{50}$ Citado en Colyvan, M., The Indispensability of Mathematics, p. 10.

${ }^{51}$ Ibid., p. 11.

${ }^{52}$ Maddy, P., "Three Forms of Naturalism", en: Shapiro, S. (ed.), The Oxford Handbook of Philosophy of Mathematics and Logic, Oxford: Oxford University Press, 2005, p. 454.
} 
de nuestra teoría... no reflejan lo que está realmente presente en una situación física dada... entonces parece razonable concluir que esas partes de nuestra teoría no han sido, de hecho, confirmadas por nuestros métodos científicos"53.

La idea es que, de acuerdo al naturalismo, debemos aceptar la práctica científica, y los científicos constantemente apelan a entidades y procesos que no toman como verdaderos; pero, de acuerdo al holismo confirmacional, las teorías se confirman como un todo. Por lo tanto, estos principios son incompatibles. Ya que el argumento de Quine depende de ambos principios, no es un argumento exitoso para justificar el realismo matemático. En palabras de Maddy: "Si nos mantenemos fieles a nuestros principios naturalistas, debemos admitir una distinción entre las partes verdaderas de una teoría y las partes que son meramente útiles. Incluso debemos admitir que algunas de estas partes útiles podrian ser de hecho indispensables, en el sentido de que una teoría sobre los mismos fenómenos no sería igualmente buena sin dichas partes. Aceptando todo esto, la indispensabilidad de las matemáticas en teorias científicas bien confirmadas no es suficiente para establecer su verdad"54.

\subsection{Argumento 4: La versión explicativa del argumento de indispensabilidad}

Una manera de distinguir entre las partes verdaderas y las partes falsas de una teoría científica es a través de las explicaciones que la teoría facilita. En la base de esta distinción se encuentra el llamado principio de "inferencia a la mejor explicación" (IME), explicado por Hartry Field en los siguientes términos: "[S]upongamos (a) que tenemos ciertas creencias, creencias sobre 'los fenómenos', y a las que no estamos dispuestos a renunciar; (b) que este tipo de 'fenómenos' en los que creemos es vasto y complejo; (c) que tenemos una muy buena explicación de estos fenómenos (entendido como un cuerpo de principios relativamente simples, no ad hoc, a partir de los cuales [dichos fenómenos] se siguen como consecuencia); y (d) una de las premisas asumidas en dicha explicación es la afirmación $\mathrm{S}$, y estamos bien convencidos de que no es posible una explicación de los mismos fenómenos que no apele a la afirmación S. La idea de 'inferencia a la mejor explicación' es que en estas circunstancias tenemos fuertes razones para creer en la afirmación S"55.

El principio IME implica que, si una afirmación es indispensable para la mejor explicación de un conjunto dado de fenómenos, entonces tenemos

\footnotetext{
53 Ibid., p. 456.

${ }^{54}$ Citado en Resnik, M., Mathematics as a Science of Patterns, p. 46.

55 Field, H., Realism, Mathematics \& Modality, Oxford: Basil Blackwell, 1989, p. 15.
} 
razones para creer en ella, "independientemente de si esa creencia es en sí misma observacional, e independientemente de si las entidades de las que se trata dicha creencia son observables"56.

La versión explicativa del AI sustituye la primera premisa de la versión de Quine por el principio IME y apela a la supuesta indispensabilidad de las matemáticas en las mejores explicaciones cientificas. De esta manera, concluye que, siguiendo el principio IME, debemos aceptar la verdad de las afirmaciones matemáticas que figuran en estas explicaciones y, por lo tanto, la existencia de los objetos matemáticos sobre los que dichas afirmaciones cuantifican. Este argumento se puede esquematizar de la manera siguiente:

P1) Debemos creer racionalmente en la existencia de cualquier entidad que sea indispensable en nuestras mejores explicaciones científicas (IME).

P2) Los objetos matemáticos son indispensables en nuestras mejores explicaciones científicas.

C) Por lo tanto, debemos creer racionalmente en la existencia de los objetos matemáticos ${ }^{57}$.

El argumento se basa en el hecho de que las mismas explicaciones que postulan objetos concretos inobservables tales como los electrones, las ondas electromagnéticas, etcétera, también postulan objetos matemáticos abstractos. Por lo tanto, en los casos en los que consideremos que una explicación es correcta, no solo tenemos razones para creer en los inobservables concretos, sino que las mismas razones justificarian creer, también, en los objetos matemáticos. Por supuesto, uno podría no creer que los electrones existan, pero la idea del AI es que existe una conexión entre la creencia en los electrones y la creencia en los objetos matemáticos.

\section{Respuesta}

Joseph Melia ${ }^{58}$ critica esta versión del AI sosteniendo que existe una distinción entre los objetos que juegan un rol representacional y los que juegan un rol estrictamente explicativo, y el principio IME se aplica solamente a estos últimos. El rol que juegan las matemáticas en la ciencia se limita, simplemente, a representar hechos concretos y a ayudar a extraer inferencias relacionadas

\footnotetext{
56 Ibid., p. 15.

57 Adaptado de Baker, A., "Mathematical Explanation in Science", p. 613.

58 Melia, J., "Weaseling Away the Indispensability Argument", en: Mind, v. CIX, n. 435 (2000), pp. 455-479; "Response to Colyvan”, en: Mind, v. CXI (2002), pp. 75-79.
} 
con dichos objetos. Esto hace que las matemáticas sean una herramienta fundamental, incluso indispensable, en la ciencia, pero esto no justifica que incluyamos a los objetos matemáticos en nuestra ontología. En palabras de Melia: "[L]as matemáticas son el andamio necesario sobre el cual el puente [de la ciencia] debe construirse. Pero una vez que el puente ha sido construido, el andamio puede retirarse"59.

De acuerdo a Melia, el rol de las matemáticas en explicaciones científicas debe entenderse como el de representar y facilitar inferencias acerca de hechos concretos que sí poseen poder explicativo. Es decir, ya que las matemáticas ofrecen buenas representaciones del mundo físico, algunas explicaciones requieren de su uso, pero esto no significa que los objetos matemáticos sean en sí mismo explicativos. Si decimos, por ejemplo, que " $F$ ha ocurrido porque $P$ mide $\sqrt{ } 2$ de largo", a pesar de que estamos mencionando al número $\sqrt{ } 2$ en la explicación, es el largo del objeto físico $\mathrm{P}$ y no el número real $\sqrt{ } 2$ que usamos para representarlo, el que realiza el trabajo explicativo ${ }^{60}$.

Esta postura representacionalista de las matemáticas es también suscrita por Bueno, quien sostiene que las matemáticas juegan en ciencia un rol representativo, un rol inferencial, y un rol expresivo. En todos los casos, para cumplir estos roles, el formalismo matemático debe ser interpretado empíricamente. En la mayoría de los casos, sin embargo, esta interpretación va a dejar de lado aspectos tanto en el mundo físico como en la estructura matemática. Si uso un círculo para representar al sol, evidentemente estoy dejando de lado varios aspectos del sol mismo, como su temperatura, y, al mismo tiempo, también dejo de lado varios aspectos del círculo, por ejemplo, su curvatura perfecta.

Ahora bien, el reto de Melia para los defensores del AI explicativo es mostrar que las matemáticas pueden jugar un rol genuinamente explicativo en las explicaciones científicas y que su rol no se limita a brindar buenas representaciones de las propiedades fisicas relevantes que figuran en explicaciones exitosas. Este reto ha conducido a que en los últimos años una nueva versión del AI explicativo tome prominencia en la filosofia de las matemáticas. Dicha versión se enfoca no en explicaciones científicas en general, sino en las llamadas explicaciones matemáticas de fenómenos fisicos donde, supuestamente, el rol de las matemáticas es genuinamente explicativo.

\footnotetext{
59 Melia, J., "Weaseling Away the Indispensability Argument", p. 469.

60 Cf., Melia, J., "Response to Colyvan", p. 76.
} 


\subsection{Argumento 5: Las explicaciones matemáticas de fenómenos físicos}

Como respuesta a Melia, Alan Baker ${ }^{61}$ y Mark Colyvan ${ }^{62}$ sostienen que existen situaciones en las que las matemáticas juegan un rol genuinamente explicativo. En estos casos, supuestamente son las matemáticas en símismas las responsables de la explicación. El argumento quedaría de la siguiente manera:

P1) Debemos creer racionalmente en la existencia de cualquier entidad que sea indispensable en nuestras mejores explicaciones científicas (IME). P2) Las matemáticas pueden jugar un rol genuinamente explicativo en ciencia.

C) Por lo tanto, debemos creer racionalmente en la existencia de los objetos matemáticos ${ }^{63}$.

Este argumento, llamado comúnmente el "Argumento de indispensabilidad ampliado", depende crucialmente de si existen genuinas explicaciones matemáticas de fenómenos físicos. Un supuesto caso, propuesto por Baker64, es la explicación de los ciclos vitales de las cigarras periódicas del género magicicada. Estos insectos emergen simultáneamente por dos semanas cada 13 o 17 años, dependiendo de la zona geográfica donde residan. La mejor explicación disponible en la literatura evolutiva para la longitud de los ciclos es que ambos ciclos son primos y es evolutivamente ventajoso para las especies biológicas desarrollar ciclos vitales primos. Esto se debe, por un lado, a que los ciclos vitales primos minimizan las posibilidades de intersección con depredadores y, también, a que los ciclos primos disminuyen las posibilidades de intersección con subespecies del ciclo vital alternativo; lo cual es ventajoso porque así se garantiza la emergencia simultánea y, consecuentemente, se maximizan las posibilidades de procreación. Ya que la propiedad primo es esencial para el funcionamiento de estas explicaciones y no puede expresarse sin usar matemáticas, es decir, no puede parafrasearse en lógica de primer orden 65 , la explicación depende de dicha propiedad. Ya que esta es la mejor explicación de la longitud de los ciclos vitales de estas cigarras de acuerdo a la literatura científica disponible -de hecho, el

61 Baker, A., "Are there Genuine Mathematical Explanations of Physical Phenomena?", en: Mind, v. CXIV (2005), pp. 223-238; "Mathematical Explanation in Science", en: British Journal for the Philosophy of Science, v. LX (2009), pp. 611-633.

62 Colyvan, M., The Indispensability of Mathematics, Oxford: Oxford University Press, 2001.

63 Baker, A., "Mathematical Explanation in Science", p. 613.

64 Baker, A., "Are there Genuine Mathematical Explanations of Physical Phenomena?", en: Mind,

v. CXIV (2005), pp. 223-238.

${ }^{65}$ Cf., Baker, A., "Mathematical Explanation in Science", p. 620. 
biólogo Robert May considera que este es el único caso de una aplicación directa de la teoría de números en biología-, para Baker esto justifica creer que los ciclos en sí mismos son primos, es decir, que poseen la propiedad matemática de ser primos. Poniendo la conclusión en términos de estructuralismo ante rem, podemos decir que los ciclos ejemplifican los números naturales 13 o 17 y, por lo tanto, comparten con ellos la propiedad de ser primos.

\section{Respuesta}

Considero, sin embargo, que este tipo de casos no son contraargumentos a la postura representacionalista de Melia y Bueno. En el caso de las cigarras, por ejemplo, tal como he argumentado en detalle en otro artículo66, los ciclos vitales en sí mismos no son primos. Por el contrario, ellos poseen una propiedad común a varios intervalos temporales. Es cierto que hubiera sido extremadamente dificil identificar esta propiedad del tiempo sin la ayuda de las matemáticas, pero en la medida en que podamos separar la propiedad física de su representación matemática, el punto de Melia sobre el rol representacional de las matemáticas sigue en pie $-\mathrm{y}$, ciertamente, podemos hacer esto en el caso de las cigarras-. Las propiedades del tiempo relevantes en esta situación pueden deducirse a partir de las nociones básicas de combinación y congruencia de intervalos temporales, las cuales son propiedades no matemáticas perfectamente aceptables y pueden fácilmente parafrasearse en lógica de primer orden. Debido a esto, los aspectos matemáticos de esta explicación sirven para representar una propiedad del tiempo, que es la propiedad relevante para los ciclos vitales. Por lo tanto, este caso no justifica la conclusión de la versión explicativa del $\mathrm{AI}^{67}$. Mi punto es que, en las llamadas explicaciones matemáticas de fenómenos físicos, tenemos una indispensabilidad práctica, pero el rol de las matemáticas es últimamente representacional.

\section{Conclusión}

Debido a que los objetos matemáticos, de existir, no tendrían poderes causales, algunos filósofos naturalistas han considerado que deben ser excluidos de nuestra ontología en virtud de que, en principio, sería imposible adquirir conocimiento acerca de dichos objetos. Sin embargo, el estructuralismo

\footnotetext{
${ }^{66}$ Barrantes, M., "Optimal Representations and the Enhanced Indispensability Argument", en: Synthese, v. CXCVI, 1 (2019), pp. 247-263.

${ }^{67}$ Para detalles $c f$. ibid.
} 
ante rem es una postura realista que explicaría, de manera compatible con el naturalismo, el descubrimiento de verdades sobre los objetos matemáticos: ya que los objetos matemáticos serian posiciones en estructuras matemáticas que pueden ser ejemplificadas en el mundo físico, podríamos tomar conocimiento de las estructuras matemáticas abstractas a través de la observación de dichas ejemplificaciones concretas. ¿Y cómo se justificaría el llamar a las estructuras físicas ejemplificaciones de estructuras matemáticas, dándoles, así, estatuto ontológico de existencia a estas últimas? Paradójicamente, el naturalismo mismo ha sido usado como justificación. El realismo matemático se justificaría por el abrumador éxito de las ciencias naturales matematizadas.

Evidentemente, existen filósofos que han puesto en duda la tesis naturalista. Otros dudan de que el éxito de las teorias científicas justifique nuestras creencias en los objetos inobservables postulados por dichas teorias. Otros, incluso, ponen en duda el éxito mismo de las teorías científicas matematizadas, y hay hasta quienes dudan de la existencia del mundo en sí o de la distinción entre realidad y ficción. Lo que he mostrado en este artículo es que, inclusive los filósofos que aceptamos ciertas versiones del naturalismo y el realismo científico, no estamos justificados en creer en la existencia de estructuras matemáticas. Mi estrategia ha sido discutir cinco argumentos que pretenden justificar el realismo matemático a partir de la aplicabilidad de las matemáticas en la ciencia y lo he hecho sin adoptar una posición explícita sobre los debates mencionados. Consideremos, por ejemplo, el argumento de indispensabilidad. Rechazar la conclusión negando la primera premisa sería conceder demasiado a los indispensabilistas, pues ellos aún podrian decir que existe un vínculo entre el realismo científico y el realismo matemático. Contrario a esto, lo que he mostrado en este artículo es que, una vez que prestamos atención a la manera como se aplican las matemáticas en ciencia, podemos ver que tal vínculo no existe: una postura no implica a la otra.

Mi discusión en este artículo no constituye un argumento positivo en favor del ficcionalismo matemático. Simplemente, sostengo que la aplicabilidad de las matemáticas en ciencia no brinda razones para adoptar el realismo matemático. Sin embargo, existen varias otras versiones del realismo matemático cuya justificación no depende de la aplicabilidad de las matemáticas en la ciencia, tales como la defensa de Cargile ${ }^{68}$ del platonismo matemático

${ }^{68}$ Cargile, “On 'Alexander's' Dictum”, en: Topoi, v. XXII, n. 2 (2003), pp. 143-149. 
puro, la justificación coherentista del estructuralismo ante rem de Shapiro69, o el platonismo autónomo de Russel Marcus ${ }^{70}$. Grandes nombres de la filosofia contemporánea fueron realistas matemáticos, incluyendo a Frege, Gödel, y Quine. El fascinante debate sobre el realismo matemático continúa.

Recibido: 04/01/2018

Aceptado: 09/10/2018

\section{Bibliografia}

Baker, A., "Are there Genuine Mathematical Explanations of Physical Phenomena?", en: Mind, n. 114, 2005, pp. 223-238. https://doi.org/10.1093/mind/fzi223

Baker, A., "Mathematical Explanation in Science", en: British Journal for the Philosophy of Science, n. 60, 2009, pp. 611-633. https://doi.org/10.1093/bjps/axp025

Bangu, S., The applicability of mathematics in science: indispensability and ontology, New York: Palgrave Macmillan, 2012.

Bangu, S., "On 'The Unreasonable Effectiveness of Mathematics in the Natural Sciences", en: Ippoliti, E. y otros (eds.), Models and Inferences in Science, Cham: Springer, 2016. https://doi.org/10.1007/978-3-319-28163-6_2

Barrantes, M., "Optimal Representations and the Enhanced Indispensability Argument", en: Synthese, v. CXCVI, n. 1, 2017, pp. 247-263. https://doi.org/10.1007/ s11229-017-1470-4

Benacerraf, P. (1973), "Mathematical Truth", en: Benacerraf, P. y H. Putnam (eds.), Philosophy of mathematics. Selected Readings, 2da edición, Cambridge: Cambridge University Press, 1973.

Bueno, O., "An Easy Road to Nominalism", en: Mind, v. CXXI, 2012, p. 484. https:// doi.org/10.1093/mind/fzs1 14

Bueno, O. y M. Colyvan, "An Inferential Conception of the Application of Mathematics", en: Noûs, v. XLV, n. 2, 2011, pp. 345-374. https://doi. org/10.1111/j.1468-0068.2010.00772.x

Bueno, O. y S. French, The Applicability of Mathematics in Science: Immersion, Inference and Interpretation, Oxford: Oxford University Press, 2018.

Cargile, S., “On 'Alexander's Dictum”, en: Topoi, v. XXII, n. 2, 2003, pp. 143-149. https:// doi.org/10.1023/A:1024926205716

Colyvan, M., The indispensability of mathematics, Oxford: Oxford University Press, 2001. https://doi.org/10.1093/019513754X.001.0001

Colyvan, M., An Introduction to the Philosophy of Mathematics, Cambridge: Cambridge University Press, 2012.

\footnotetext{
${ }^{69}$ Shapiro, S., Philosophy of Mathematics. Structure and Ontology, Nueva York: Oxford University Press, 1997.

${ }^{70}$ Marcus, R., Autonomy Platonism and the Indispensability Argument, Lanham: Lexington Books, 2015.
}

ARETÉ Revista de Filosofía, vol. XXXI, Nº 1, 2019 / ISSN 1016-913X 
Davies, P., The Mind of God, Londres: Penguin Book, 1992.

Field, H., Realism, Mathematics \& Modality, Oxford: Basil Blackwell, 1989.

Humphreys, P., Extending Ourselves, Oxford: Oxford University Press, 2004. https:// doi.org/10.1093/0195158709.001.0001

Lange, M., "What Makes a Scientific Explanation Distinctively Mathematical?", en: British Journal for the Philosophy of Science, v. LXIV, n. 3, 2013, pp. 485-511. https:// doi.org/10.1093/bjps/axs012

Lyon, A., "Mathematical Explanations Of Empirical Facts, And Mathematical Realism", en: Australasian Journal of Philosophy, v. XC, n. 3, 2011, pp. 559-578. https:// doi.org/10.1080/00048402.2011.596216

MacBride, F., "Can Ante Rem Structuralism solve the Access Problem?", The Philosophical Quarterly, v. LVIII, n. 230, 2008, p. 58. https://doi. org/10.1111/j.1467-9213.2007.524.x

Maddy, P., "Three forms of Naturalism", en: Shapiro, S. (ed.), The Oxford Handbook of Philosophy of Mathematics and Logic, Oxford: Oxford University Press, 2005. https://doi.org/10.1093/0195148770.003.0013

Marcus, R., Autonomy Platonism and the Indispensability Argument, Lanham: Lexington Books, 2015

Melia, J., "Weaseling Away the Indispensability Argument", en: Mind, v. CIX, n. 435, 2000, pp. 455-479 https://doi.org/10.1093/mind/109.435.455

Melia, J., "Response to Colyvan", en: Mind, n. 111, 2002, pp. 75-79. https://doi. org/10.1093/mind/111.441.75

Penrose, R., The Emperor's New Mind: Concerning Computers, Minds and the Laws of Physics. Londres: Vintage, 1990. https://doi.org/10.1119/1.16207

Resnik, M., Mathematics as a Science of Patterns, Oxford: Oxford University Press, 1997.

Resnik, M., "Quine and the Web of Belief", en: Shapiro, S. (ed.), The Oxford Handbook of Philosophy of Mathematics and Logic, Oxford: Oxford University Press, 2005. https://doi.org/10.1093/0195148770.003.0012

Shapiro, S., Philosophy of mathematics. Structure and ontology, Nueva York: Oxford University Press, 1997.

Shapiro, S., Thinking about mathematics. The Philosophy of Mathematics, Oxford: Oxford University Press, 2001.

Shapiro, S., "Philosophy of Mathematics and its Logic. Introduction", en: Shapiro, S. (ed.), The Oxford Handbook of Philosophy of Mathematics and Logic, Oxford: Oxford University Press, 2005. https://doi.org/10.1093/0195148770.001.0001

Shapiro, S. (ed.), The Oxford Handbook of Philosophy of Mathematics and Logic, Oxford: Oxford University Press, 2005. https://doi.org/10.1093/0195148770.001.0001

Suppes, P., "Models of Data", en: Nagel, E. y otros (eds.), Logic, Methodology, and Philosophy of Science: Proceedings of the 1960 International Congress, Stanford: Stanford University Press, 1962.

Wigner, E., "The Unreasonable Effectiveness of Mathematics in the Natural Sciences", en: Communications on Pure and Applied Mathematics, n. 13, 1960, pp. 1-14. https://doi.org/10.1002/cpa.3160130102 